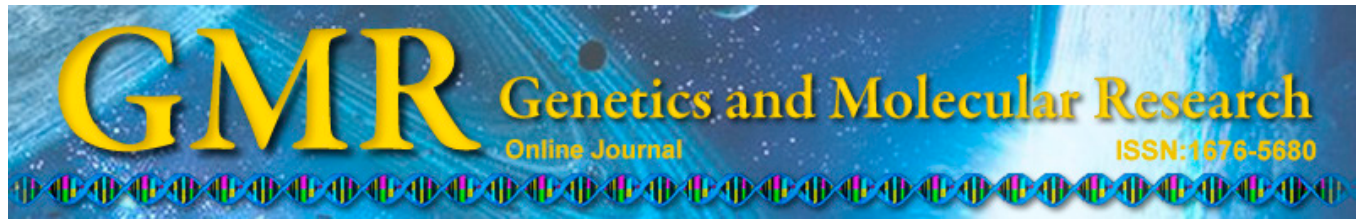

\title{
Immunohistochemical subtypes of diffuse large B-cell lymphoma in the head and neck region
}

\author{
J.M. Li, J. Hou, L. Li and Y. Wang \\ Department of Pathology, Sichuan Cancer Hospital, Chengdu, China \\ Corresponding author: J.M. Li \\ E-mail: lijiman_ljm@126.com \\ Genet. Mol. Res. 14 (2): 3889-3896 (2015) \\ Received October 15, 2014 \\ Accepted January 19, 2015 \\ Published April 27, 2015 \\ DOI http://dx.doi.org/10.4238/2015.April.27.3
}

\begin{abstract}
The objectives of this study were to detect immunohistochemical subtypes of diffuse large B-cell lymphoma (DLBCL) of the head and neck, to compare the Hans, Choi, and Tally algorithms and to examine the significance of protein expression in these algorithms. This study included 103 DLBCL patients at Sichuan Cancer Hospital between May 2010 and October 2012. Immunohistochemistry was performed for CD10, B-cell lymphoma 6 protein (Bcl-6), mutated melanoma-associated antigen 1 (MUM1), germinal center B-cell-expressed transcript 1 (GCET1), forkhead box protein P1 (FOXP1), and LIM domain only 2 (LMO2). Subtypes were determined according to the Hans, Choi, and Tally algorithms. Positive staining for CD10 was detected in 16 patients $(15.53 \%)$, for Bcl-6 in 68 patients $(66.02 \%)$, for MUM1 in 69 patients $(66.99 \%)$, for GCET1 in 21 patients $(20.39 \%)$, for FOXP1 in 75 patients $(72.82 \%)$, and for LMO2 in 50 patients $(48.54 \%)$. The Hans algorithm identified 26 patients $(25.2 \%)$ with the germinal center B-cell (GCB) subtype and 77 (74.8\%) with the activated B-cell (ABC) subtype. In the Choi algorithm, 25 patients $(24.3 \%)$ were identified with the GCB subtype and $78(75.7 \%)$ with the ABC subtype. In the Tally algorithm, 20 patients (19.4\%) had the GCB subtype and 83 (80.6\%)
\end{abstract}


had the ABC subtype. Expression of CD10, MUM1, GCET1, FOXP1, and LMO2 correlated with algorithm $(\mathrm{P}<0.05)$; however, Bcl-6 did not correlate with the Hans and Choi algorithms. DLBCL of the head and neck is most commonly the ABC subtype, not GCB. The Hans, Choi, and Tally algorithms were not significantly different.

Key words: Diffuse large B-cell lymphoma; Head and neck region; Immunohistochemical subtypes

\section{INTRODUCTION}

Diffuse large B-cell lymphoma (DLBCL) is the most common type of non-Hodgkin lymphoma. DLBCL can be grouped into two subtypes based on differences in gene expression profiles: germinal center B-cell (GCB) type and activated B-cell (ABC) type. Treatment and prognosis are completely different for these subtypes (Alizadeh et al., 2000). Determination of gene expression profiles requires fresh or frozen tissue for extraction of sufficient RNA, which is costly and difficult to implement in clinical practice and retrospective studies. Instead of utilizing expensive tests such as gene chips for treatment or diagnosis, many researchers have proposed the use of immunohistochemistry with a few antibodies that represent different Bcell differentiation stages, which is an easier and more economical method for classification, guiding treatment, and predicting prognosis of DLBCL. Various immunohistochemical classification methods have been reported. The goal of our study was to compare the classic Choi and Hans algorithms and the new Tally algorithm that was reported in 2011, and to examine each immunohistochemical index with the different algorithms.

\section{MATERIAL AND METHODS}

\section{General information}

We identified all 126 patients with an original diagnosis of DLBCL in the Pathology Department of Sichuan Cancer Hospital from May 2010 to October 2012. Twenty-three patients were excluded because they were either consultation cases or the results were less accurate because very little tissue was obtained. Therefore, 103 patients were included and were in accordance with the World Health Organization diagnostic criteria for hematopoietic and lymphoid tissue tumors. Among the 103 patients, 65 were men and 38 were women, with a male to female ratio of 1.71 . The median age was 61 years, and the mean age was $59.81 \pm 15.15$ years. There were 11 patients with tumors in the nasal cavity and nasopharynx, 10 with tumors in the tonsillar region, 3 with tumors in the submaxillary region, 1 with a tumor in the postcricoid area, 4 with tumors in the thyroid gland, 57 with tumors in the cervical lymph node area, 6 with tumors in the oropharyngeal region, 2 with tumors on the face, 2 with tumors on the parotid glands, 1 with a tumor in the maxillary sinus, and 6 with tumors on the root of the tongue.

\section{Methodology}

Immunohistochemistry (EnVision method; Dako, Glostrup, Denmark) was used to detect the expression status (positive or negative) of CD10, B-cell lymphoma 6 protein (Bcl-6), 
mutated melanoma-associated antigen 1 (MUM1), germinal center B-cell-expressed transcript 1 (GCET1), forkhead box protein P1 (FOXP1), and LIM domain only 2 (LMO2). Information about primary antibodies (name, clone number, source, and specificity) is displayed in Table 1. Positive and negative controls were included for all immunohistochemically stained samples. The criteria for positive staining was $\geq 80 \%$ positively stained cells for the GCET1, MUM1, and FOXP1 antibodies, and $\geq 30 \%$ positively stained cells for the other antibodies including CD10, Bcl-6, and LMO2.

\section{Table 1. Primary antibody specifications.}

\begin{tabular}{llll}
\hline Antibody name & Company and catalog No. & Dilution & Positive staining pattern \\
\hline CD10 (common antigen of acute lymphoblastic leukemia) & ZA-0526 & Ready-to-use & Cell membrane \\
Bcl-6 (B-cell lymphoma 6 protein) & MAB-0598 & Ready-to-use & Cell nucleus \\
MUM1 (mutated melanoma-associated antigen 1) & ZM-0399 & Ready-to-use & Cell nucleus \\
FOXP1 (forkhead box protein P1) & ZM-0354 & Ready-to-use & Cell nucleus \\
GCET1 (germinal center B cell-expressed transcript 1) & ZM-0369 & Ready-to-use & Cell membrane \\
LMO2 (LIM domain only 2) & ZA-0572 & Ready-to-use & Cell nucleus \\
\hline
\end{tabular}

\section{Classification criteria}

Specific classification standards for the 3 algorithms are shown in Figure 1 (Hans et al., 2004), Figure 2 (Choi et al., 2009), and Table 2 (Meyer et al., 2011).

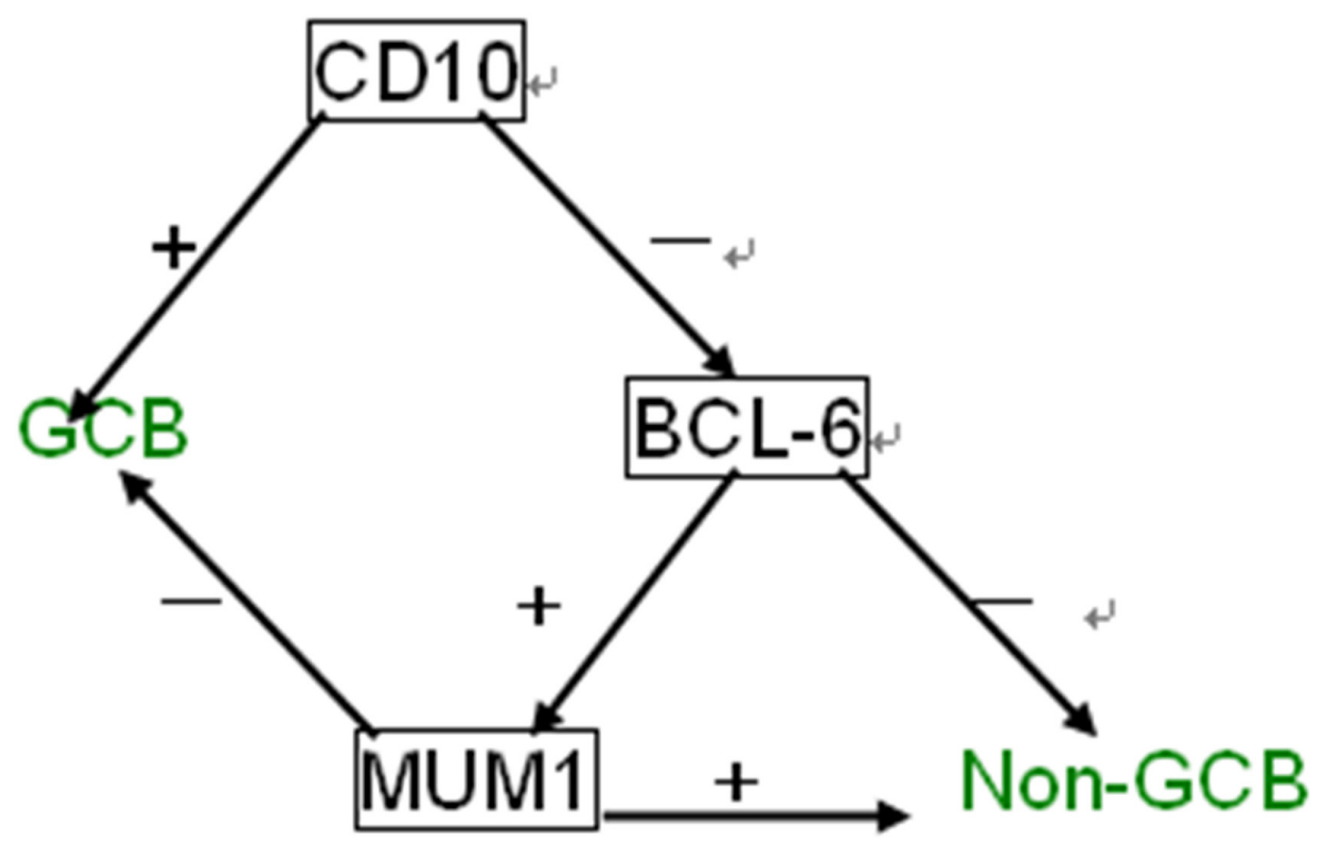

Figure 1. Hans algorithm. 


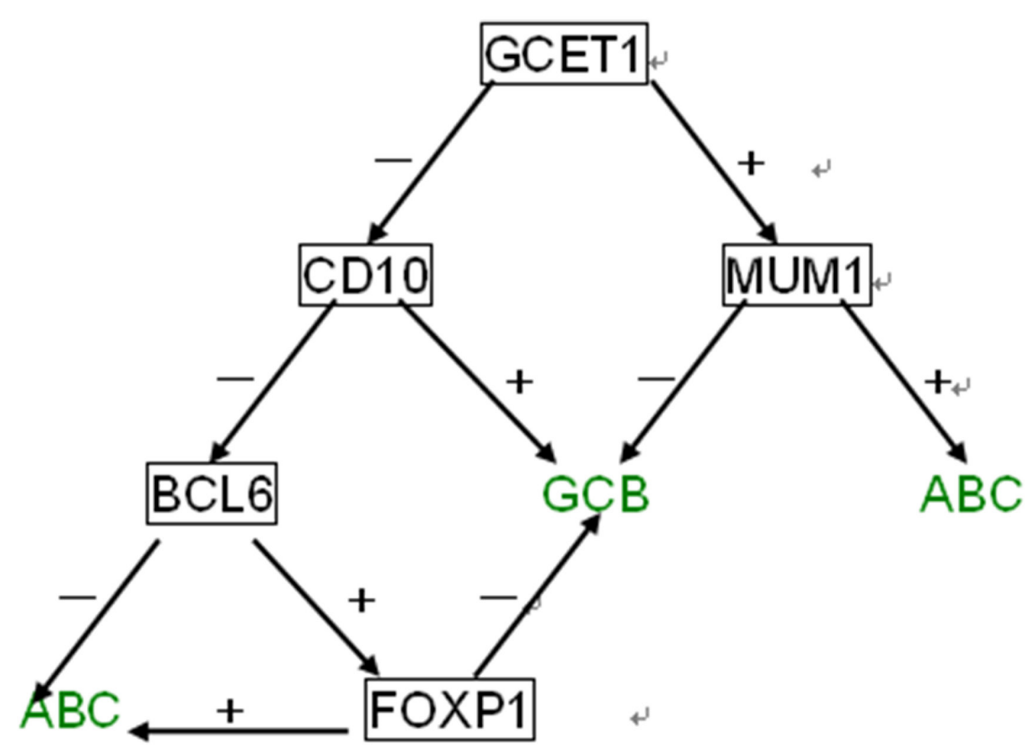

Figure 2. Choi algorithm.

\section{Table 2. Tally algorithm.}

\begin{tabular}{llc}
\hline & GCB score & ABC score \\
\hline CD10 $(-/+)$ & $0 / 1$ & \\
GCET1 $(-/+)$ & $0 / 1$ & $0 / 1$ \\
MUM1 $(-/+)$ & & $0 / 1$ \\
FOXP1 $(-/+)$ & & GCB score $>$ ABC score suggests GCB type; GCB score $<$ ABC score suggests ABC type; for \\
Total & cases where GCB $=$ ABC, LMO2 should also be considered (1 point added to GCB score if \\
& LMO2-positive). \\
\hline
\end{tabular}

ABC, activated B cell; FOXP1, forkhead box protein P1; GCB, germinal center B cell; GCET1, germinal center B cell-expressed transcript 1; LMO2, LIM domain only 2; MUM1, mutated melanoma-associated antigen 1.

\section{Statistical analysis}

Data were analyzed using the SPSS software, version 19.0 (IBM Corp., Armonk, NY, USA). Chi-square $\left(\chi^{2}\right)$ tests were used to compare the 3 different algorithms.

Four-fold table $\chi^{2}$ tests for regulation were performed with a significance level of $\alpha=$ 0.05 and a theoretical value $<5$. The Fisher exact probability tests were performed with theoretical values $<1$. Moreover, Kappa values were calculated for pairwise comparisons of the 3 algorithms to evaluate their consistency.

\section{RESULTS}

\section{Histomorphological results}

There was diffuse proliferation and infiltration of tumor cells, and necrosis, hemorrhaging, and inflammatory cell infiltration were also observed (Figure 3). The tumor cells had 
little cytoplasm and large round or oval nuclei with single or multiple nucleoli. In some tumor tissues, the tumor cells had lost their regular vesicular nuclear form, and they were squeezed into a spindle formation because of compression during sample collection. It was often more difficult to determine whether these deformed tumor cells were derived from centroblasts in the germinal center or activated immunoblasts.

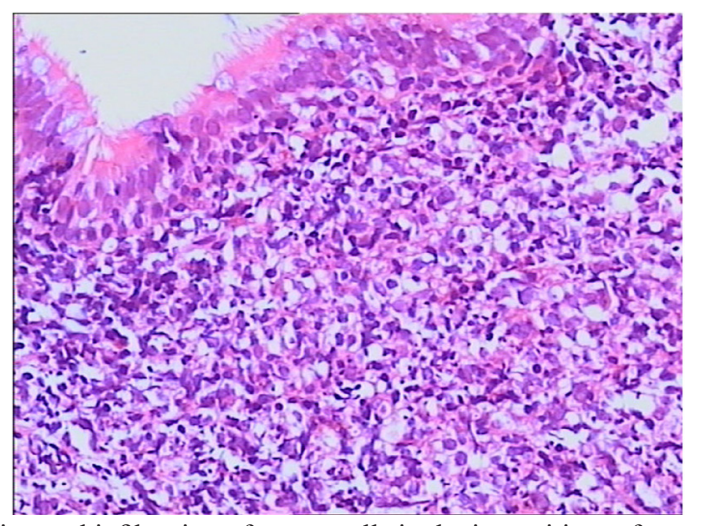

Figure 3. Diffuse proliferation and infiltration of tumor cells in the interstitium of nasopharynx (HE; 200X).

\section{Immunophenotype of the samples}

Immunohistochemistry in all 103 samples yielded positivity rates for $\mathrm{CD} 10, \mathrm{Bcl}-6$, MUM1, GCET1, FOXP1, and LMO2 of 15.53\% (16/103), 66.02\% (68/103), 66.99\% (69/103), $20.39 \%(21 / 103), 72.82 \%$ (75/103), and 48.54\% (50/103), respectively (Figure 4). Expression status (positive or negative) for CD10, MUM1, GCET1, FOXP1, and LMO2 was statistically significant for the diagnostic results of the classification; however, Bcl-6 expression status was not significant in either the Choi or the Hans algorithm. For a pairwise comparison of the 3 algorithms, Kappa values were calculated, and there were no significant differences between any 2 of the 3 algorithms $(\mathrm{P}>0.05$; Tables 3 and 4$)$.
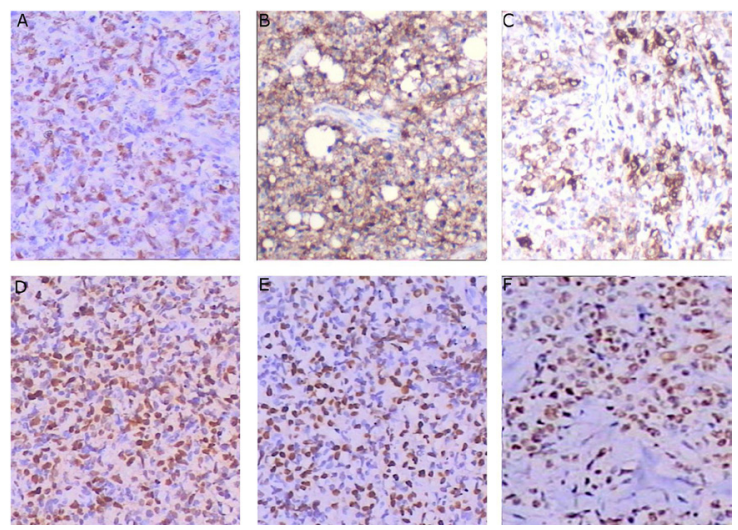

Figure 4. Immunohistochemistry results of tumor cells EnVision100X. A: Bcl-6 positive in cell nucleus, B: CD10 positive on cell membrane, C: GCET1 positive on cell membrane, D: MUM1 positive in cell nucleus, E: FOXP1 positive in cell nucleus, $\mathbf{F}$ : LMO2 positive in cell nucleus. 
Table 3. Correlations between protein expression and the 3 algorithms.

\begin{tabular}{|c|c|c|c|c|c|c|c|c|c|c|c|c|c|}
\hline & & \multicolumn{4}{|c|}{ Hans algorithm } & \multicolumn{4}{|c|}{ Choi algorithm } & \multicolumn{4}{|c|}{ Tally algorithm } \\
\hline & & GCB & $\mathrm{ABC}$ & $\chi^{2}$ value & $P$ value & $\mathrm{GCB}$ & $\mathrm{ABC}$ & $\chi^{2}$ value & $P$ value & $\mathrm{GCB}$ & $\mathrm{ABC}$ & $\chi^{2}$ value & $P$ value \\
\hline \multicolumn{14}{|l|}{ CD10 } \\
\hline$(+)$ & 16 & 14 & 2 & 35.099 & 0.000 & 12 & 4 & 23.353 & 0.000 & 9 & 7 & 16.424 & 0.000 \\
\hline$(-)$ & 87 & 12 & 75 & & & 13 & 74 & & & 11 & 76 & & \\
\hline \multicolumn{14}{|l|}{ Bcl-6 } \\
\hline$(+)$ & 68 & 20 & 48 & 1.843 & 0.175 & 19 & 49 & 1.466 & 0.226 & - & - & - & - \\
\hline$(-)$ & 35 & 6 & 29 & & & 6 & 29 & & & & & & \\
\hline \multicolumn{14}{|c|}{ MUM1 } \\
\hline$(+)$ & 69 & 6 & 63 & 30.329 & 0.000 & 6 & 63 & 27.591 & 0.000 & 5 & 64 & 19.789 & 0.000 \\
\hline$(-)$ & 34 & 20 & 14 & & & 19 & 15 & & & 15 & 19 & & \\
\hline \multicolumn{14}{|c|}{ FOXP1 } \\
\hline$(+)$ & 75 & - & - & - & - & 12 & 63 & 10.270 & 0.001 & 5 & 70 & 28.667 & 0.000 \\
\hline$(-)$ & 28 & & & & & 13 & 15 & & & 15 & 13 & & \\
\hline \multicolumn{14}{|c|}{ GCET1 } \\
\hline$(+)$ & 21 & - & - & - & - & 11 & 10 & 11.339 & 0.001 & 13 & 8 & 27.117 & 0.000 \\
\hline$(-)$ & 82 & & & & & 14 & 68 & & & 7 & 75 & & \\
\hline \multicolumn{14}{|l|}{ LMO2 } \\
\hline$(+)$ & 50 & - & - & - & - & - & - & - & - & 20 & 30 & 26.053 & 0.000 \\
\hline$(-)$ & 53 & & & & & & & & & 0 & 53 & & \\
\hline
\end{tabular}

ABC, activated B cell; Bcl-6, B cell lymphoma 6 protein; FOXP1, forkhead box protein P1; GCB, germinal center B cell; GCET1, germinal center B cell-expressed transcript 1; LMO2, LIM domain only 2; MUM1, mutated melanoma-associated antigen 1.

Table 4. Pairwise comparisons of the 3 algorithms.

\begin{tabular}{lccc}
\hline & Hans and Choi & Choi and Tally & Hans and Tally \\
\hline Kappa value & 0.765 & 0.462 & 0.554 \\
P value & 0.074 & 0.104 & 0.098 \\
\hline
\end{tabular}

\section{DISCUSSION}

Alizadeh et al. (2000) divided B-cells of different differentiation stages into 2 groups based on gene expression profiles: GCB type, which specifically express CD10, Bcl-6, and LMO-2; and $\mathrm{ABC}$ type or non-germinal center type (non-GC type) cells, which express FOXP1, cyclin D2, among others. These 2 DLBCL subtypes showed differences in response and prognosis after treatment with the same chemotherapy regimens: the 5-year survival rate of the patients with the GCB subtype was significantly higher than that of the patients with the $\mathrm{ABC}$ subtype (76 vs 16\%). However, gene expression profiling requires fresh or frozen tissue, and is costly, which has limited its application in the clinic. Therefore, several researchers have proposed using a few antibodies that represent different B-cell differentiation stages for immunohistochemical staining in order to easily and economically classify DLBCL. In 2004, Hans et al. (2004) proposed a commonly used algorithm in which DLBCL is classified as either GCB or non-GCB type based on the expression status of CD10, Bcl-6, and MUM1. The concordance rate of the Hans algorithm and gene expression profiling is approximately $80 \%$. The selected markers include CD10, a common antigen in acute lymphoblastic leukemia that is considered an indicator of germinal center cell origin, and Bcl-6, which is expressed on germinal center B-cells and CD4-positive T-cells and may indicate lymphomatous origin. MUM1, a member of the interferon regulatory factor family, is expressed in approximately $70 \%$ of 
DLBCL cases, most of which are immunoblasts or activated B-cells. Using these 3 indicators, 26 cases in our study were classified as the GCB subtype and 77 as the ABC subtype. In 2009, 2 additional indicators were added to the Hans algorithm by Choi as an improvement to the classic method (Choi et al., 2009): GCET1 (Montes-Moreno et al., 2008) and FOXP1 (Banham et al., 2001). This new algorithm still classified DLBCL as either GCB or ABC subtype, and yielded results that were $93 \%$ consistent with gene expression profiling. The newly added indicator GCET1, which is encoded by the gene transcription factor 1, is expressed on and is a marker of germinal center B-cells. On the other hand, FOXP1 is a B-cell activation marker that is necessary during B-cell development. Based on the Choi algorithm, our study included 25 cases of GCB subtype and 78 cases of ABC subtype disease. Several new algorithms for classification have been proposed, such as the Natkunam and Nyman algorithms (Natkunam et al., 2008; Nyman et al., 2009). The latest algorithm is the Tally algorithm, proposed by Meyer et al. (2011), which uses LMO2, a marker of germinal center B-cells, in place of Bcl-6 from the Choi algorithm. Notably, the Tally algorithm was found to be $98 \%$ consistent with gene expression profiling. In our study, 17 cases were the GCB subtype and 83 cases were the ABC subtype, according to the Tally algorithm. There is currently no consensus about how DLBCL should be classified, and algorithms vary in different pathology departments. According to the Hans algorithm, cases are classified as GCB subtype if they are CD10-positive, regardless of whether MUM1 and Bcl-6 are positive, which decreases the importance of these 2 indicators to some extent and likely increases the proportion of cases classified as the GCB subtype. In our study, 2 CD10-positive cases were categorized as the GCB subtype by the Hans algorithm and the $\mathrm{ABC}$ subtype by the Choi algorithm; 6 of these cases were classified as the $\mathrm{ABC}$ subtype by the Tally algorithm. Moreover, 68 cases in our study were positive for Bcl-6 as a marker of the germinal center. However, only 20 of these 68 cases were categorized as the GCB subtype by the Hans algorithm, and 19 of these cases were classified as the GCB by the Choi algorithm. Expression of Bcl-6 is reportedly somewhat unstable (positivity rate ranging from 29.6-100\%; Yin and Li, 2007) and differs with the use of an immunohistochemical staining enhancer (Min et al., 2012). Because of the poor reproducibility of Bcl-6, it was replaced by LMO2 in the Tally algorithm as an improvement on the Choi and Hans algorithms. In the present study, we performed a $\chi^{2}$ test and demonstrated that the Bcl- 6 expression is irrelevant for classification in the Hans and Choi algorithms $(\mathrm{P}>0.05)$. Our study did not include gene chip technology, so it is not possible to reveal the relationship between Bcl-6 expression and gene chip classification results.

Our study found that DLBCL in the nasopharynx was mostly ABC subtype. This is consistent with a previous study in a Chinese population that had found that DLBCL of the ABC subtype was most common (Wang et al., 2007). Our study found no significant differences in the pairwise comparisons of the 3 algorithms. However, in clinical practice, these 3 algorithms each have distinct advantages and disadvantages. For example, the Hans algorithm is relatively simple and economical, but it overemphasis of the importance of CD10 may lead to increased classification as the GCB subtype. The Choi algorithm requires at least 2 indicators for classification, lowering the importance of CD10 and Bcl-6 and making it more objective than the Hans algorithm. However, both the Hans and Choi algorithms use the path method during classification, so some determinations may be based on only 1 or 2 positive indicators, reducing the effect of the other indicators. Alternatively, the scoring method used by the Tally algorithm ensures that various indicators enter the scoring process in parallel, making this method relatively objective. Notably, the involvement of more indicators has made it more 
expensive than previous methods. In our study, we demonstrated that differential expression of CD10, MUM1, GCET1, FOXP1, and LMO2 were all significantly important for classification, suggesting that the Tally algorithm, which includes all 5 of these indicators, is a more accurate and practical method for DLBCL classification. Further evaluation is necessary in future studies that include a large number of cases and report comparisons with gene chip results.

\title{
ACKNOWLEDGMENTS
}

\author{
ment (\#110269).
}

Research supported by the Research Projects of the Sichuan Provincial Health Depart-

\section{REFERENCES}

Alizadeh AA, Eisen MB, Davis RE, Ma C, et al. (2000). Distinct types of diffuse large B-cell lymphoma identified by gene expression profiling. Nature 403: 503-511.

Banham AH, Beasley N, Campo E, Fernandez PL, et al. (2001). The FOXP1 winged helix transcription factor is a novel candidate tumor suppressor gene on chromosome 3p. Cancer Res. 61: 8820-8829.

Choi WW, Weisenburger DD, Greiner TC, Piris MA, et al. (2009). A new immunostain algorithm classifies diffuse large B-cell lymphoma into molecular subtypes with high accuracy. Clin. Cancer Res. 15: 5494-5502.

Hans CP, Weisenburger DD, Greiner TC, Gascoyne RD, et al. (2004). Confirmation of the molecular classification of diffuse large B-cell lymphoma by immunohistochemistry using a tissue microarray. Blood 103: 275-282.

Meyer PN, Fu K, Greiner TC, Smith LM, et a1. (2011). Immunohistochemical methods for predicting cell of origin and survival in patients with diffuse large B-cell lymphoma treated with rituximab. J. Clin. Oncol. 29: 200-207.

Min M, Lin L, Bi CF, Wang XQ, et al. (2012). Analysis of the immunohistochemical subtypes and prognosis of primary diffuse large B-cell lymphoma of the central nervous system. Zhonghua Zhong Liu Za Zhi. 34: 110-116.

Montes-Moreno S, Roncador G, Maestre L, Martinez N, et al. (2008). Gcetl (centerin), a highly restricted marker for a subset of germinal center-derived lymphomas. Blood 111: 351-358.

Natkunam Y, Farinha P, Hsi ED, Hans CP, et al. (2008). LMO2 protein expression predicts survival in patients with diffuse large B-cell lymphoma treated with anthracycline-based chemotherapy with and without rituximab. J. Clin. Oncol. 26: 447-454

Nyman H, Jerkeman M, Karjalainen-Lindsberg ML, Banham AH, et al. (2009). Prognostic impact of activated B-cell focused classification in diffuse large B-cell lymphoma patients treated with R-CHOP. Mod. Pathol. 22: 1094-1101.

Wang J, Ke XY, Zhao LZ, Li M, et al. (2007). Analysis of prognostic factors in 74 cases of diffuse large B-cell lymphoma. Zhonghua Xue Ye Xue Za Zhi 28: 667-670.

Yin HF and Li T (2007). Comparative study of heterogeneity of extranodal and nodal diffuse large B-cell lymphoma. Beijing Da Xue Xue Bao 39: 158-162. 